Canad. Math. Bull. Vol. 60 (4), 2017 pp. 736-746

http://dx.doi.org/10.4153/CMB-2017-007-x

(C) Canadian Mathematical Society 2017

\title{
Levi's Problem for Pseudoconvex Homogeneous Manifolds
}

To the memory of R. Remmert

Bruce Gilligan

Abstract. Suppose $G$ is a connected complex Lie group and $H$ is a closed complex subgroup. Then there exists a closed complex subgroup $J$ of $G$ containing $H$ such that the fibration $\pi: G / H \rightarrow$ $G / J$ is the holomorphic reduction of $G / H$; i.e., $G / J$ is holomorphically separable and $\mathcal{O}(G / H) \cong$ $\pi^{\star} \mathcal{O}(G / J)$. In this paper we prove that if $G / H$ is pseudoconvex, i.e., if $G / H$ admits a continuous plurisubharmonic exhaustion function, then $G / J$ is Stein and $J / H$ has no non-constant holomorphic functions.

\section{Introduction}

The original Levi problem dealt with the characterization of domains of holomorphy in $\mathbb{C}^{n}$ having smooth boundary in terms of conditions on that boundary. Grauert [11] (resp. Narasimhan [22]) showed that a complex manifold (resp. complex space) that admits a smooth (resp. continuous) strictly plurisubharmonic exhaustion function is Stein. However, Grauert also pointed out that there exist pseudoconvex domains in tori all of whose holomorphic functions are constant; see e.g., [23]. This shows that the Levi problem for pseudoconvex domains that are not strongly pseudoconvex fails, in general.

In this paper we restrict our attention to complex homogeneous manifolds $X:=$ $G / H$ with $G$ a connected complex Lie group and $H$ a closed complex subgroup, and we seek conditions under which its holomorphic function algebra $\mathcal{O}(G / H)$ is Stein. Setting

$$
J:=\{g \in G \mid f(g H)=f(e H) \text { for all } f \in \mathcal{O}(G / H)\}
$$

yields a closed complex subgroup of $G$ containing $H$, and, consequently, one has the holomorphic fibration $\pi: G / H \rightarrow G / J, g H \mapsto g J$, which we call the holomorphic reduction of $G / H$ [9]. By construction, one has $\mathcal{O}(G / H) \cong \pi^{*} \mathcal{O}(G / J)$. The question then reduces to considerations of when the holomorphically separable complex homogeneous manifold $G / J$ is Stein.

Now it is known that holomorphic separability implies Steinness for complex Lie groups [19], for complex nilmanifolds [9], and for complex solvmanifolds [14]. But the situation is much different when the group acting is semisimple or reductive. For

Received by the editors September 19, 2016; revised January 31, 2017.

Published electronically April 3, 2017.

This work was partially supported by an NSERC Discovery Grant.

AMS subject classification: 32M10, 32U10, 32A10, 32Q28.

Keywords: complex homogeneous manifold, plurisubharmonic exhaustion function, holomorphic reduction, Stein manifold, Remmert reduction, Hirschowitz annihilator. 
example, $\mathbb{C}^{n} \backslash\{0\}$ is not Stein whenever $n>1$. What is known for $G$ reductive is the following: $G / H$ holomorphically separable implies $H$ algebraic [5] and $G / H$ is Stein if and only if $H$ is reductive $[18,24]$. A reductive complex Lie group is the complexification of a totally real maximal compact subgroup and the compactness of this subgroup is playing an essential role. An excellent survey of the function theory on $G / H$ for $G$ reductive can be found in [1, Chapter 5], and we refer the interested reader to that book and the references listed therein.

This paper analyzes the holomorphic function theory of pseudoconvex complex homogeneous manifolds, where a complex manifold is pseudoconvex if it admits a continuous plurisubharmonic exhaustion function. In [10, Main Theorem] we proved that the base of the holomorphic reduction of any pseudoconvex complex homogeneous manifold $G / H$ is Stein and its fiber has no non-constant holomorphic functions if $G$ is solvable (see also [10, Theorem 8.5$]$ ) or if $G$ is reductive (see also [10, Theorem 7.5]).

Here we prove that this result holds more generally for pseudoconvex homogeneous manifolds of mixed groups, where by a mixed group we mean that $G$ is a connected, simply connected, complex Lie group that has a Levi-Malcev decomposition $G=S \ltimes R$, where $R$ is the radical of $G, S$ is a maximal semisimple subgroup, and both $\operatorname{dim} R>0$ and $\operatorname{dim} S>0$.

Theorem 1.1 Let $G$ be a connected complex Lie group and $H$ a closed complex subgroup of $G$ such that $G / H$ is pseudoconvex. Suppose $G / H \rightarrow G / J$ is the holomorphic reduction of $G / H$. Then $G / J$ is Stein and $\mathcal{O}(J / H)=\mathbb{C}$.

This note is organized as follows. Section 2 contains some technical results that are needed for the proof. Section 3 presents the proof of Theorem 1.1.

\section{Technical Preparations}

\subsection{The Hirschowitz Annihilator}

Every element $\xi \in \mathfrak{g}$, the Lie algebra of $G$, can be thought of as a right invariant vector field on $G$ and, as such, pushes down to a holomorphic vector field $\xi_{X}$ on any complex homogeneous manifold $X:=G / H$ of the group $G$. An inner integral curve in such a homogeneous space $X$ is a non-constant holomorphic map $\mathbb{C} \rightarrow X$ with relatively compact image in $X$ that is the integral curve of some vector field $\xi_{X}$ assigned to some $\xi \in \mathfrak{g}$. Hirschowitz [12] considered such concepts in the context of infinitesimally homogeneous manifolds, where a manifold is infinitesimally homogeneous if every tangent space is generated by global holomorphic vector fields. A complex manifold that is homogeneous under the action of a Lie group of holomorphic transformations is infinitesimally homogeneous.

Hirschowitz showed that a pseudoconvex, infinitesimally homogeneous $X$ that does not contain any inner integral curve is Stein [12, Proposition 3.4]. This is the starting point of our investigations of pseudoconvex homogeneous manifolds that are not Stein given in [10]. By the maximum principle, any plurisubharmonic function on a complex manifold $X$ is constant along every inner integral curve in $X$. One has 
to determine the "directions of degeneracy" of plurisubharmonic functions in terms of a certain subset of $\mathfrak{g}$ whose corresponding holomorphic vector fields "kill them". So in [10] we define the Hirschowitz annihilator $\mathcal{A}$ to be the connected Lie subgroup of $G$ whose Lie algebra is given by

$$
\mathfrak{a}:=\left\{\xi \in \mathfrak{g} \mid \xi_{X} \varphi\left(x_{0}\right)=0, \forall \varphi \in \mathscr{P}(X)\right\},
$$

where $\mathscr{P}(X)$ is the space of continuous plurisubharmonic functions on $X:=G / H$. For continuous functions, the derivative is understood in the sense of distributions. By definition $\mathfrak{a}$ is a complex vector subspace of $\mathfrak{g}$, and it is also a Lie subalgebra of $\mathfrak{g}$ that properly contains $\mathfrak{h}$, if $X$ is not Stein [10, Lemma 3.3]. The corresponding (not necessarily closed) connected complex subgroup $\mathcal{A}$ of $G$ contains the identity component $H^{0}$ of $H$ and defines a complex foliation $\mathscr{F}=\left\{F_{x}\right\}_{x \in X}$, the Levi foliation of the manifold $X$. Every leaf $F_{x}$ of this foliation is a relatively compact immersed complex submanifold of $X$ containing every inner integral curve in $X$ passing through the point $x$ and is an orbit of the group $\mathcal{A}$. In general, complex foliations are rather difficult to understand. But here the foliation arises from a subgroup strongly reflecting the underlying geometry of the homogeneous manifold and is related to the existence of a plurisubharmonic exhaustion on it. This allows a sufficiently good understanding in order to analyze the structure from the point of view of its holomorphic function algebra.

If the leaves of the foliation are closed, then they are compact and $X$ is holomorphically convex [10, Theorem 4.1]. Indeed, the holomorphic reduction is then given by the Remmert reduction [25] and has compact fiber and Stein base. The main difficulty occurs when this is not the case. The following observation is essential in what follows.

Remark 2.1 A question of Serre [26] asks whether the total space of every holomorphic fibration with Stein fiber and Stein base is itself Stein. Counterexamples are known, some of which are even homogeneous, e.g., [7]. Among other things, Lemma 2.2 asserts that Serre's question has an affirmative answer in our setting.

Lemma 2.2 ([12] and [10, Remark 2.5]) Suppose $Y$ is a pseudoconvex complex homogeneous manifold that admits a holomorphic fibration $Y \stackrel{F}{\rightarrow} B$ with both $F$ and $B$ being holomorphically separable manifolds. Then $Y$ is Stein.

Proof Note that every complex homogeneous manifold is infinitesimally homogeneous. As neither $F$ nor $B$ can contain any inner integral curve, the same is true of $Y$. Therefore, $Y$ is Stein [12, Proposition 3.4].

\subsection{Tits' Normalizer Fibration}

Suppose $H$ is a $k$-dimensional closed subgroup of an $n$-dimensional Lie group $G$. The Lie algebra $\mathfrak{h}$ of $H$ is a Lie subalgebra of $\mathfrak{g}$ and can be considered as a point in the Grassman manifold $G r(k, n)$ of $k$-dimensional vector subspaces of $\mathfrak{g}$. Since $\operatorname{ad}(G) \subset$ $G L(\mathfrak{g})$, there is a natural action of $\operatorname{ad}(G)$ on $G r(k, n)$, and the $\operatorname{ad}(G)$-orbit of the point $\mathfrak{h}$ can be identified with $G / N$, where $N:=N_{G}\left(H^{0}\right)$, i.e., the normalizer in $G$ of the identity component $H^{0}$ of $H$. Via the Plücker embedding, $G r(k, n)$ can be 
realized as a submanifold of some projective space such that the automorphisms of the Grassman are restrictions of those automorphisms of the projective space that stabilize the embedded Grassman. In this way, we realize $G$ acting linearly on $G / N$ via the adjoint representation. Since $H \subset N_{G}\left(H^{0}\right)$, we have the Tits normalizer fibration $G / H \rightarrow G / N$ [27].

Remark 2.3 Let $I$ be a complex Lie subgroup of the complex Lie group G. The normalizer $N_{G}\left(I^{0}\right)$, where $I^{0}$ denotes the connected component of the identity of $I$ is a closed subgroup of $G$, since it is the isotropy subgroup of a point in a complex projective space under the adjoint action of the group $G$.

\subsection{Closure of Orbits}

Let $G$ be a (real) Lie group and $H$ a closed subgroup of $G$. For $I$ a normal Lie subgroup of $G$, set $F:=\operatorname{cl}_{X}\left(I . x_{0}\right)$, where $x_{0}$ is the base point of $X:=G / H$.

Lemma 2.4 Let $J:=\operatorname{cl}_{G}(I \cdot H)$. Then $J$ is a subgroup of $\operatorname{Stab}_{G}(F)$.

Proof Since $F$ is closed, $\operatorname{Stab}_{G}(F)$ is closed. Clearly, $I \cdot H \subset \operatorname{Stab}_{G}(F)$, so $J \subset$ $\operatorname{Stab}_{G}(F)$.

Remark 2.5 As a consequence, $F=J . x_{0}$. Now for $x \in X$ define $[x]:=\operatorname{cl}_{X}(I . x)$ and note that if $x=g\left(x_{0}\right)$, then $[x]=g(F)$. Thus, the classes $[x]$ are the fibers of the homogeneous fibration $G / H \rightarrow G / J$.

\subsection{Fibrations Over Projective Orbits}

We use the following notation for the derived series of the Lie group $G$ :

$$
G^{(0)}:=G, G^{(1)}:=G^{\prime}=[G, G], \ldots, G^{(k)}:=\left[G^{(k-1)}, G^{(k-1)}\right] \text { for all } k>1 .
$$

Lemma 2.6 Let $X:=G / H$ be an orbit of a connected complex Lie group $G$ acting holomorphically and effectively on some projective space. Assume that $J$ is a connected, complex subgroup of $G$ that has positive dimensional orbits in $G / H$ that are relatively compact. Then $J$ is a subgroup of every subgroup $G^{(m)}$ of the derived series of $G$.

Proof By a result of Chevalley [6], the image of the commutator group $G^{\prime}$ in the automorphism group of the ambient projective space is algebraically closed. This means that $G^{\prime}$ is acting as an algebraic group on the projective space, and, in particular, that its orbits in $G / H$ are closed. Thus, one has the commutator fibration $G / H \rightarrow G / H \cdot G^{\prime}$. Let $x_{0} \in G / H$ be the base point. The Stein Abelian group $\bar{G} / \bar{G}_{x_{0}} \cdot \bar{G}^{\prime}$ contains $G / H \cdot G^{\prime}$ as a $G$-orbit [13], where the bar denotes the Zariski closure. Therefore, J. $x_{0}$ is contained in the fiber of $G / H \rightarrow G / H \cdot G^{\prime}$ by the maximum principle with $J . x_{0}$ still being relatively compact in the closed fiber $G^{\prime} / H \cap G^{\prime}$. Replace $G$ (resp. $H$ ) by $G^{(1)}:=G^{\prime}$ (resp. $H^{(1)}:=H \cap G^{\prime}=G_{x_{0}}^{\prime}$ ) and iterate the argument to see that $J$ is a subgroup of every group in the derived series of $G$. 
Lemma 2.7 Let $G$ be a connected complex Lie group, $H$ a closed complex subgroup of $G$, and I a closed complex subgroup of $G$ containing $H$ with $G / I$ equivariantly embedded in the complex projective space $\mathbb{P}_{N}$. If $J$ is a connected, normal, complex Lie subgroup of $G$ whose orbits in $G / H$ are relatively compact and cover its orbits in $G / I$, then the $J$-orbits in $G / H$ are flag manifolds.

Proof By Lemma 2.6, the image of $J$ in the automorphism group of $G / I$ lies in the image of every subgroup $G^{(m)}$ of the derived series of $G$. Since $G$ has finite dimension, one has $G^{(k)}=\left(G^{(k)}\right)^{\prime}=G^{(k+1)}=\cdots$ for some $k$. As the $J$-orbits have positive dimension, $G^{(k)}$ is a positive dimensional perfect Lie group that is acting algebraically on $\mathbb{P}_{N}$. Its radical $R_{G^{(k)}}$ is nilpotent [15]. Since $J$ is normal, its radical $R_{J}=R_{G^{(k)}} \cap J$ [15]. Thus, $R_{J}$ is a connected complex subgroup of the unipotent group $R_{G^{(k)}}$ and so is acting algebraically on $\mathbb{P}_{N}$ with each of its orbits a closed copy of $\mathbb{C}^{q}$ for some $q \geq 0$. Since the $J$-orbits are relatively compact, we must have $q=0$. This implies that $R_{J}$ acts trivially, and thus $J$ is acting algebraically as a semisimple group on $G / I$.

Now $A:=\operatorname{cl}_{G}(J \cdot H) \subset K:=\operatorname{cl}_{G}(J \cdot I)$, since $H \subset I$ by assumption. By Remark 2.5 and the assumption that the $J$-orbits are relatively compact, we get the diagram

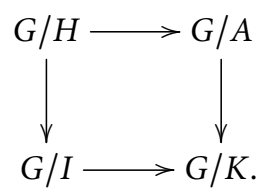

The $J$-orbit through the base point in $G / I$ is contained in $K / I$, and the latter space is compact. Thus, the closure of this $J$-orbit lies in $K / I$. Since $J$ is acting algebraically as a semisimple complex group, this orbit is Zariski open in its closure, and its boundary consists of $J$-orbits of strictly lower dimension. Since $J \triangleleft G$, all orbits have the same dimension, and so the boundary is empty; i.e., the $J$-orbits are closed and thus compact. Compact orbits of a complex Lie group acting holomorphically on a projective space are flag manifolds. Since flag manifolds are simply connected and the $J$-orbits in $G / H$ cover the $J$-orbits in $G / I$, it follows that the $J$-orbits in $G / H$ are flag manifolds.

Corollary 2.8 Let $G$ be a connected complex Lie group, $H$ a closed complex subgroup of $G$, and I a closed complex subgroup of $G$ containing $H$ with $G / I$ equivariantly embedded in the complex projective space $\mathbb{P}_{N}$ and the fibers of the fibration $G / H \rightarrow G / I$ holomorphically separable. If $J$ is a connected, normal, complex Lie subgroup of $G$ whose orbits in $G / H$ are relatively compact, then the J-orbits in $G / H$ are flag manifolds.

Proof Since $I / H$ is holomorphically separable, the $J$-orbits intersect the fibers of the fibration $G / H \rightarrow G / I$ transversally and so cover the corresponding $J$-orbits in $G / I$, which are necessarily relatively compact in $G / I$. The result now follows from Lemma 2.7 . 


\subsection{Normality Under Closure and Complexification}

For $J$ a connected Lie subgroup of $G$ the complexification $J^{\mathbb{C}}$ of $J$ is the connected Lie subgroup of $G$ corresponding to the Lie algebra $j+i j$, where $j$ denotes the Lie algebra of $J$. Subsequently, we need to consider what happens to normality under closure and complexification, and so the following lemma is important.

Lemma 2.9 Suppose I is a connected normal Lie subgroup of a connected Lie subgroup $J$ of a connected complex Lie group $G$. Then

(i) $I \triangleleft \mathrm{cl}_{G}(J)$,

(ii) $I^{\mathbb{C}} \triangleleft J^{\mathbb{C}}$.

In particular, $I^{\mathbb{C}} \triangleleft \widetilde{J}$, where $\widetilde{J}$ is the smallest connected closed complex subgroup of $G$ that contains $J$.

Proof (i) If $\lim g_{n}=g \in \mathrm{cl}_{G}(J)$, where $g_{n} \in J$, then $g I g^{-1}=\lim g_{n} I g_{n}^{-1} \subset I$.

(ii) This follows from the fact that $[\mathfrak{i}, \mathfrak{j}] \subset \mathfrak{i}$ implies $[\mathfrak{i}+i \mathfrak{i}, \mathfrak{j}+i \mathfrak{i j}] \subset \mathfrak{i}+i \mathfrak{i}$.

Note that $\widetilde{J}$ can be formed by alternately taking the complexification and closure of $J$. Applying (i) and (ii), as appropriate, completes the proof.

\subsection{Existence of a Fibration by a Solvmanifold}

Proposition 2.10 (Personal communication from K. Oeljeklaus) Let $X:=G / \Gamma$, where $\Gamma$ is a discrete subgroup of a connected, simply connected, complex Lie group $G$ with Levi decomposition $G=S \ltimes R$ and $\operatorname{dim} R>0$. Then there is a connected, complex, solvable subgroup $H$ of $G$ normalized by $\Gamma$, containing $R$, with $H \cdot \Gamma$ a closed subgroup of $G$. In particular, one has the proper fibration (unless $S=\{e\}$ )

$$
G / \Gamma \longrightarrow G / H \cdot \Gamma=S / S \cap H \cdot \Gamma
$$

that has the connected complex solvmanifold $H / H \cap \Gamma$ as its typical fiber.

Proof If the $R$-orbits themselves are closed, set $H:=R$. If not, then the Zassenhaus Lemma [2] is used in [8, Theorem 2] to show the existence of a minimal connected complex solvable subgroup $H_{1} \subset G$ normalized by $\Gamma$ and containing the identity component of $\operatorname{cl}_{G}(R \cdot \Gamma)$. If $H_{1} \cdot \Gamma$ is closed in $G$, one has the desired result with $H:=H_{1}$.

Otherwise, let $N_{1}:=N_{G}\left(H_{1}\right)$. Since $\Gamma$ normalizes the identity component $N_{1}^{0}$ of $N_{1}$, it also normalizes its radical $R_{1}$. Now $H_{1} \subset R_{1}$, because $H_{1}$ is solvable and normal in $N_{1}$. Either $R_{1} \cdot \Gamma$ is closed in $G$, or the identity component of $\mathrm{cl}_{G}\left(R_{1} \cdot \Gamma\right)$ is contained in $N_{1}^{0}$. Applying the Zassenhaus Lemma again (in $N_{1}^{0}$ ), we see that this identity component is solvable and normalized by $\Gamma$, since $N_{1}^{0} \cdot \Gamma=N_{1}$ is closed in $G$. Let $\mathrm{H}_{2}$ be the smallest connected closed complex subgroup of $G$ that contains this identity component. Then $\mathrm{H}_{2}$ is solvable, normalized by $\Gamma$, and its dimension is strictly greater than the dimension of $H_{1}$. A finite number of steps yields the desired connected complex solvable group $H$. 


\subsection{Existence of a Tower}

Lemma 2.11 Suppose $\Gamma$ is a cocompact, discrete subgroup of a (positive dimensional) connected solvable Lie group $L$ such that $L^{\mathbb{C}} / \Gamma$ is Stein, where $L^{\mathbb{C}}$ is the complexification of $L$. Then there exists a fibration by the center $Z$ of the nilradical of $L^{\mathbb{C}}$

$$
L^{\mathbb{C}} / \Gamma \stackrel{\left(\mathbb{C}^{*}\right)^{k}}{\longrightarrow} L^{\mathbb{C}} / Z \cdot \Gamma
$$

that has $\left(\mathbb{C}^{*}\right)^{k}$ as fiber with $k>0$.

Proof Let $N_{0}$ be the nilradical of $L$ and $N$ the nilradical of $L^{\mathbb{C}}$. Then $N_{0}$ has closed orbits in $L / \Gamma$ by a theorem of Mostow [20] or [21], and thus $N$ has closed orbits in $L^{\mathbb{C}} / \Gamma$. Let $Z$ be the center of $N$ (resp. $Z_{0}$ of $N_{0}$ ). Incidentally, note that $\operatorname{dim} Z>0$ [17]. Since $N / N \cap \Gamma$ is a closed complex submanifold of the Stein manifold $L^{\mathbb{C}} / \Gamma$, we see that $N / N \cap \Gamma$ is Stein. Hence the subgroup $Z \cdot \Gamma$ is closed by a result of Barth-Otte [4]; see also [9, Theorem 4]. Therefore, we have the fibration

$$
L^{\mathbb{C}} / \Gamma \longrightarrow L^{\mathbb{C}} / Z \cdot \Gamma \text {. }
$$

It follows that the fibers of the fibration above are $\left(\mathbb{C}^{*}\right)^{k}$-orbits for some positive integer $k$; see [9, Theorem 7].

Definition 2.12 A $\mathbb{C}^{*}$ power tower of length one is simply the manifold $\left(\mathbb{C}^{*}\right)^{p}$ for some positive integer $p$. For any integer $n>1$, a $\mathbb{C}^{*}$ power tower of length $n$ is a $\left(\mathbb{C}^{*}\right)^{k}$-bundle over a $\mathbb{C}^{*}$ power tower of length $n-1$.

Remark 2.13 Repeated application of Lemma 2.11 shows that the space $L^{\mathbb{C}} / \Gamma$ is a $\mathbb{C}^{*}$ power tower of length $n$ for some positive integer $n$.

\section{Proof of the Main Result}

\subsection{Formulation of the Strategy of the Proof}

Remark 3.1 There is a technical point that can arise in our construction, in that an intermediary fibration whose fiber is not connected might be involved. This is handled by a type of Stein factorization for homogeneous fibrations. Suppose $G$ is a connected Lie group that contains a closed subgroup $I$ containing a closed subgroup $H$. Let $\widetilde{I}$ be those connected components of $I$ that meet $H$. Then $\widetilde{I}$ is a closed subgroup of $G$ containing $H$, and the fibration $G / H \rightarrow G / \widetilde{I}$ has connected fiber $\widetilde{I} / H$.

Strategy of the Proof Assume $G / H$ is a pseudoconvex homogeneous manifold that is not Stein. In order to prove the theorem we construct a closed complex subgroup $I$ of $G$ containing $H$ with $\operatorname{dim} I>\operatorname{dim} H$ and $I / H$ connected, possibly with the aid of Remark 3.1, such that

(a) $\mathcal{O}(I / H)=\mathbb{C}$ and

(b) every continuous plurisubharmonic exhaustion function on $G / H$ induces a continuous plurisubharmonic exhaustion function on $G / I$. 
Then if $G / H \rightarrow G / J$ is the holomorphic reduction, $I$ is a subgroup of $J$ because of (a), $G / I$ is pseudoconvex because of (b) and is either Stein (then $I=J$ and we are done) or not Stein, and one applies the construction until one does reach the holomorphic reduction; see also the last paragraph of the proof below for more details.

Remark 3.2 Here is a list of some complex homogeneous manifolds that do satisfy (b), whenever they occur as the fiber of a homogeneous fibration $G / H \rightarrow G / I$. For (ii) and (iii), the basic tool to prove this is Kiselman's Minimum Principle [16, Theorem 2.2]:

(i) compact complex homogeneous manifolds,

(ii) Cousin groups (see [10, Lemma 6.1(1)]),

(iii) the fibers of certain $\mathbb{C}^{*}$ power towers provided the exhaustion function is constant on the underlying circle power tower; see below.

\subsection{The Proof Itself}

Proof Let $G / H$ be a non-Stein pseudoconvex homogeneous manifold. Then its Hirschowitz annihilator $\mathcal{A}$ satisfies $\operatorname{dim} \mathcal{A}>\operatorname{dim} H$. We define subgroups by setting

(a) $G_{1}:=N_{G}(\mathcal{A})$; note that $H$ is a subgroup of $N_{G}(\mathcal{A})$ by $[10$, p. 42] and

(b) $G_{2}:=N_{G_{1}}\left(H^{0}\right)$.

Note that in (a), because the $\mathcal{A}$-orbits are positive dimensional and relatively compact, the fibration $G / H \rightarrow G / N_{G}(\mathcal{A})$ is not a covering and its fiber is not Stein. Also because of the fact that $\mathcal{A}$ is normal in $G_{1}$, if the fibration $G_{1} / H \rightarrow G_{1} / N_{G_{1}}\left(H^{0}\right)$ is a covering (resp. has a Stein fiber), then the $\mathcal{A}$-orbits in $G_{1} / H$ are flag manifolds by Lemma 2.7 (resp. by Corollary 2.8). We are done, since setting $I:=\mathcal{A} \cdot H$ yields a fibration $G / H \rightarrow G / I$ with $I / H$ compact and thus satisfying (i) and (ii). Thus, we need only consider the setting where $G_{2} / H$ is a non-Stein pseudoconvex homogeneous manifold. If the Hirschowitz annihilator $\mathcal{A}_{2}$ for $G_{2} / H$ is not normal in $G_{2}$, we apply (a) again setting $G_{3}:=N_{G_{2}}\left(\mathcal{A}_{2}\right)$. So $G_{3}=N_{G_{3}}\left(\mathcal{A}_{2}\right)<N_{G_{1}}\left(H^{0}\right)$, the latter because $G_{3}$ is a subgroup of $G_{2}$. Now set $\widehat{G}:=G_{3} / H^{0}, \widehat{\mathcal{A}}:=\mathcal{A}_{2} / H^{0}$ and $\Gamma:=H / H^{0}$ and note that $\widehat{\mathcal{A}} \triangleleft \widehat{G}$ and has positive dimensional orbits in $\widehat{G} / \Gamma$. Furthermore, we can assume that $\widehat{G}$ is not solvable (resp. semisimple) because of [10, Theorem 8.5] (resp. [10, Theorem 7.1]), since each of these settings directly yields the desired subgroup I satisfying (i) and (ii); in the first case it arises from a fibration by a Cousin group and in the second by a compact complex manifold. Hence, we reduce to the case where $\widehat{G}$ is a mixed group. The rest of the construction below produces a subgroup $\widehat{I}$ of $\widehat{G}$ containing $\Gamma$ with $\widehat{I} / \Gamma$ satisfying (i) and (ii). Taking the preimage $I$ of $\widehat{I}$ via the quotient homomorphism $G_{3} \rightarrow G_{3} / H^{0}$ gives us the desired fibration $G / H \rightarrow G / I$. For notational convenience we suppress the hats from now on and write $G$ instead of $\widehat{G}$, etc.

Since $\mathcal{A} \triangleleft G$, we can apply Lemma 2.4 and Remark 2.5. Set $L:=\operatorname{cl}_{G}(\mathcal{A} \cdot \Gamma)$ and let $\widetilde{L}$ be the smallest connected, closed, complex subgroup of $G$ that contains $L$. Since the $\mathcal{A}$-orbits are relatively compact, $L / \Gamma$ is compact. As a consequence, $\mathcal{O}(\widetilde{L} / \Gamma)=\mathbb{C}$ by the maximum and identity principles. We claim that we can further reduce to the setting where the group $L$ has a positive dimensional radical $R_{L}$, a fact that we will use later. If $\widetilde{L}$ is semisimple, then one has the fibration $G / \Gamma \rightarrow G / \widetilde{L}$ and $\widetilde{L} / \Gamma$ is 
pseudoconvex and thus holomorphically convex [10, Theorem 7.1]. This implies that $\widetilde{L} / \Gamma$ is compact, and we are again done with $I:=\widetilde{L}$. In particular, in the rest of the proof we assume that $\operatorname{dim} R_{L}>0$.

We need to show that $\widetilde{L} / \Gamma$ satisfies (ii) in this setting. By Proposition 2.10 there is a fibration

$$
\widetilde{L} / \Gamma \longrightarrow \widetilde{L} / \widetilde{H} \cdot \Gamma
$$

with $\widetilde{H} \cdot \Gamma$ closed in $\widetilde{L}$, where $\widetilde{H}$ is a connected, solvable, complex Lie group that contains the radical $R_{\widetilde{L}}$ of $\widetilde{L}$ and is normalized by $\Gamma$. Now we claim that we can further reduce to the setting where the fiber $\widetilde{H} \cdot \Gamma / \Gamma$ of the above fibration is Stein. Otherwise, $\widetilde{H} \cdot \Gamma / \Gamma$ would be a connected, pseudoconvex solvmanifold that is not Stein and there would exist a closed complex subgroup $I$ of $\widetilde{H} \cdot \Gamma$ containing $\Gamma$ with $I / \Gamma$ a positive dimensional Cousin group [10, Theorem 8.5]. Clearly, $I / \Gamma$ satisfies conditions (i) and (ii). So from here on we can assume that $\widetilde{H} \cdot \Gamma / \Gamma$ is Stein.

Now in order to finish the proof that $\widetilde{L} / \Gamma$ satisfies (ii), we have to analyze the structure of (at least part of) the intersection of the compact orbit $L / \Gamma$ with the Stein orbit $\widetilde{H} / \widetilde{H} \cap \Gamma$. Consider the connected, real, solvable group $B:=(L \cap \widetilde{H})^{0}$. Since $R_{L} \subset R_{\widetilde{L}} \subset \widetilde{H}$ by Lemma 2.9 and $R_{L} \subset L$, it follows that $R_{L} \subset B$ and thus $\operatorname{dim} B>0$. Let $B^{\mathbb{C}}$ be its complexification. We have $\Gamma \subset N_{\widetilde{L}}\left(B^{\mathbb{C}}\right)$, since $\Gamma \subset L$ and $\Gamma$ normalizes $\widetilde{H}$ by Proposition 2.10 , and thus we can consider the fibration $\widetilde{L} / \Gamma \rightarrow \widetilde{L} / N_{\widetilde{L}}\left(B^{\mathbb{C}}\right)$. Now we can assume that $N_{\widetilde{L}}\left(B^{\mathbb{C}}\right) / \Gamma$ is not Stein, for, otherwise, $\mathcal{A}$ would have compact orbits by Corollary 2.8, a case that could be easily handled, as above. The Hirschowitz annihilator for the space $N_{\widetilde{L}}\left(B^{\mathbb{C}}\right) / \Gamma$ need not be normal in $\widetilde{L}$. So we begin the proof again with the space $N_{\widetilde{L}}\left(B^{\mathbb{C}}\right) / \Gamma$ and run at most a finite number of times (because $\operatorname{dim} G / H<\infty)$ through all of its steps until the only situation demanding further attention occurs when $N_{\widetilde{L}}\left(B^{\mathbb{C}}\right)=\widetilde{L}$. Hence, we can assume that $B^{\mathbb{C}}$ is a connected, normal, solvable subgroup of $\widetilde{L}$. As a consequence, $B^{\mathbb{C}} \cap L$ is normal in $L$ and this implies that $B \subset R_{L}$. But $R_{L} \subset B$ and so $B=R_{L}$. We claim that the $R_{L}$-orbits in $\widetilde{L} / \Gamma$ are compact. As noted above, $R_{L} \subset R_{\widetilde{L}}$ by Lemma 2.9. So by the construction of $\widetilde{H}$, one has

$$
\left(R_{L} \cdot \Gamma\right)^{0} \subset \operatorname{cl}_{\widetilde{L}}\left(R_{L} \cdot \Gamma\right)^{0} \subset((L \cap \widetilde{H}) \cdot \Gamma)^{0}=\left(R_{L} \cdot \Gamma\right)^{0},
$$

where, as usual, the superscript denotes the connected component of the identity. It follows that the $R_{L}$-orbits are closed, and since the $L$-orbits are compact, the $R_{L}$-orbits are thus also compact. Note that if $\mathfrak{r}_{L} \cap i \mathfrak{r}_{L} \neq(0)$, then the connected complex Lie group corresponding to the complex ideal $\mathfrak{r}_{L} \cap \mathfrak{i r}_{L}$ has positive dimensional orbits in the compact $R_{L}$-orbits in the $\widetilde{H}$-orbits. By the maximum principle, this contradicts our reduction to the setting where the $\widetilde{H}$-orbits are Stein. Thus, one must have $\mathfrak{r}_{L} \cap$ $i \mathfrak{r}_{L}=(0)$, and the $R_{L}$-orbits in $\widetilde{L} / \Gamma$ are totally real. Now consider the complexification $R_{L}^{\mathbb{C}}$ of $R_{L}$ that has Lie algebra $\mathfrak{r}_{L}^{\mathbb{C}}:=\mathfrak{r}_{L} \oplus i \mathfrak{r}_{L}$. According to a conjecture of Mostow [20] that was proved by Auslander-Tolimieri [3] and Mostow [21], every solvmanifold has the structure of a (real) vector bundle over a compact solvmanifold. In general, the compact base is homogeneous with respect to a group that is not a subgroup of the original solvable Lie group acting on the manifold, but rather lies in a certain algebraic hull of that group. We claim that our setting is special in that $R_{L} \subset R_{L}^{\mathbb{C}}$ can be taken 
to be that subgroup. Suppose

$$
R_{L}^{\mathbb{C}} / R_{L}^{\mathbb{C}} \cap \Gamma \stackrel{\mathbb{R}^{k}}{\longrightarrow} M
$$

is the vector bundle given by Mostow's conjecture. Since $R_{L}^{\mathbb{C}} / R_{L}^{\mathbb{C}} \cap \Gamma$ is Stein, it follows from Serre's homology condition [26] that $\operatorname{dim}_{\mathbb{C}} R_{L}^{\mathbb{C}} \geq \operatorname{dim}_{\mathbb{R}} M \geq \operatorname{dim}_{\mathbb{R}} R_{L}$. On the other hand, we have $\operatorname{dim}_{\mathbb{R}} R_{L}=\operatorname{dim}_{\mathbb{C}} R_{L}^{\mathbb{C}}$, as noted above. As a consequence, the compact base $M$ is diffeomorphic to $R_{L} / R_{L} \cap \Gamma$ and $R_{L}^{\mathbb{C}} \cap \Gamma=R_{L} \cap \Gamma$. So the $R_{L}^{\mathbb{C}}$-orbits are closed in $\widetilde{L} / \Gamma$. We can now apply Lemma 2.11 to the triple $\left(R_{L} \cap \Gamma, R_{L}, R_{L}^{\mathbb{C}}\right)$.

Now we have the fibration $\widetilde{L} / \Gamma \rightarrow \widetilde{L} / R_{L}^{\mathbb{C}} \cdot \Gamma$. Any continuous plurisubharmonic exhaustion function $\varphi$ on $G / \Gamma$ is constant on each of the $\mathcal{A}$-orbits, since these orbits lie in the level sets of the exhaustion function. By continuity, $\varphi$ is then constant on the orbits of the closure $L$ and thus on the orbits of its radical $R_{L}$. The $\left(S^{1}\right)^{k}$-orbits that arise in fibration

$$
R_{L}^{\mathbb{C}} /\left(R_{L}^{\mathbb{C}} \cap \Gamma\right) \stackrel{\left(\mathbb{C}^{*}\right)^{k}}{\longrightarrow} R_{L}^{\mathbb{C}} / Z \cdot\left(R_{L}^{\mathbb{C}} \cap \Gamma\right)
$$

given by Lemma 2.11 are part of the $R_{L}$-orbits. Thus, $\varphi$ is constant on the $\left(S^{1}\right)^{k}$-orbits.

As in [10, Lemma 6.1(2)] one can apply Kiselman's minimum principle [16, Theorem 2.2] and $\varphi$ induces a continuous plurisubharmonic function on $\widetilde{L} / Z \cdot \Gamma$. As a consequence, $\widetilde{L} / Z \cdot \Gamma$ is pseudoconvex. We continue in this fashion until a maximal semisimple group is acting transitively on the resulting quotient space $Y$. But then $Y$ is pseudoconvex and thus holomorphically convex $[10$, Theorem 7.1]. Since $\mathcal{O}(\widetilde{L} / \Gamma)=\mathbb{C}$, it follows that $Y$ is compact and we see that $\widetilde{L} / \Gamma$ satisfies (ii). This completes the proof that $\widetilde{L} / \Gamma$ satisfies (i) and (ii).

In order to complete the proof of the theorem, we assume that $G / H$ is a pseudoconvex homogeneous manifold that is not Stein with $\mathcal{O}(G / H) \neq \mathbb{C}$, and we let $G / H \rightarrow G / J$ be its holomorphic reduction. Note that $J / H$ cannot be Stein, since this would imply that $G / H$ itself would be Stein by Lemma 2.2 . We now choose the maximal $I$ given by the construction above. Then $\mathcal{O}(I / H)=\mathbb{C}$. Moreover, $G / I$ is pseudoconvex due to the fact that $I$ satisfies (ii). If $G / I$ were not Stein, then there would exist a subgroup $I_{1}$ with $\operatorname{dim} I_{1}>\operatorname{dim} I$ with $I_{1} / I$ satisfying conditions (i) and (ii). But this would imply that $I_{1} / H$ also satisfies these two conditions, contradicting the maximality of $I$. Consequently, $I=J$ and shows that the holomorphic reduction has the desired properties.

Acknowledgments We thank K. Oeljeklaus for pointing out Proposition 2.10. We also thank A. Huckleberry for stimulating discussions concerning this paper, C. Miebach for his constructive criticisms, and the referee for some further suggestions.

\section{References}

[1] D. N. Akhiezer, Lie group actions in complex analysis. Aspects of Mathematics, E27, Friedr. Vieweg \& Sohn, Braunschweig, 1995 . http://dx.doi.org/10.1007/978-3-322-80267-5

[2] L. Auslander, On radicals of discrete subgroups of Lie groups. Amer. J. Math. 85(1963), 145-150. http://dx.doi.org/10.2307/2373206

[3] L. Auslander and R. Tolimieri, On a conjecture of G. D. Mostow and the structure solvmanifolds. Bull. Amer. Math. Soc. 75(1969), 1330-1333. http://dx.doi.org/10.1090/S0002-9904-1969-12416-X 
[4] W. Barth and M. Otte, Über fast-uniforme Untergruppen komplexer Liegruppen und auflösbare komplexe Mannigfaltigkeiten. Comment. Math. Helv. 44(1969), 269-281. http://dx.doi.org/10.1007/BF02564528

[5] — Invariante holomorphe Funktionen auf reduktiven Liegruppen. Math. Ann. 201(1973), 97-112. http://dx.doi.org/10.1007/BF01359787

[6] C. Chevalley, Théorie des groupes de Lie. Tome II. Groupes algébriques. Actualités Sci. Ind., 1152, Hermann \& Cie., Paris, 1951.

[7] G. Coeuré and J.-J. Loeb, A counterexample to the Serre problem with a bounded domain of $\mathbb{C}^{2}$ as fiber. Ann. of Math. (2) 122(1985), no. 2, 329-334. http://dx.doi.org/10.2307/1971305

[8] B. Gilligan, Ends of complex homogeneous manifolds having nonconstant holomorphic functions. Arch. Math. (Basel) 37(1981), no. 6, 544-555. http://dx.doi.org/10.1007/BF01234393

[9] B. Gilligan and A. T. Huckleberry, On non-compact complex nil-manifolds. Math. Ann. 238(1978), no. 1, 39-49. http://dx.doi.org/10.1007/BF01351452

[10] B. Gilligan, C. Miebach, and K. Oeljeklaus, Pseudoconvex domains spread over complex homogeneous manifolds. Manuscripta Math. 142(2013),35-59. http://dx.doi.org/10.1007/s00229-012-0592-8

[11] H. Grauert, On Levi's problem and the imbedding of real-analytic manifolds. Ann. of Math. 68(1958), 460-472. http://dx.doi.org/10.2307/1970257

[12] A. Hirschowitz, Le problème de Lévi pour les espaces homogènes. Bull. Soc. Math. France 103(1975), no. 2, 191-201.

[13] A. T. Huckleberry and E. Oeljeklaus, Homogeneous spaces from a complex analytic view-point. In: Manifolds and Lie Groups (Notre Dame, Ind., 1980) Progr. Math., 14, Birkhäuser, Boston, MA, 1981, pp. 159-186.

[14] $\longrightarrow$ On holomorphically separable complex solv-manifolds. Ann. Inst. Fourier (Grenoble) 36(1986), no. 3, 57-65. http://dx.doi.org/10.5802/aif.1059

[15] N. Jacobson, Lie algebras. Interscience Tracts in Pure and Applied Mathematics, 10, Interscience Publishers (a division of John Wiley \& Sons), New York-London 1962.

[16] C. O. Kiselman, The partial Legendre transformation for plurisubharmonic functions. Invent. Math. 49(1978), 137-148. http://dx.doi.org/10.1007/BF01403083

[17] Y. Matsushima, On the discrete subgroups and homogeneous spaces of nilpotent Lie groups. Nagoya Math. J. 2(1951), 95-110. http://dx.doi.org/10.1017/S0027763000010096

[18] $\longrightarrow$ Espaces homogènes de Stein des groupes de Lie complexes. Nagoya Math. J. 16(1960), 205-218. http://dx.doi.org/10.1017/S0027763000007662

[19] Y. Matsushima and A. Morimoto, Sur certains espaces fibrés holomorphes sur une variété de Stein. Bull. Soc. Math. France 88(1960), 137-155.

[20] G. D. Mostow, Factor spaces of solvable groups. Ann. of Math. (2) 60(1954), 1-27. http://dx.doi.org/10.2307/1969700

[21] $\longrightarrow$ Some applications of representative functions to solvmanifolds. Amer. J. Math. 93(1971), 11-32. http://dx.doi.org/10.2307/2373444

[22] R. Narasimhan, The Levi problem for complex spaces. I, II, Math. Ann. 142(1961), 355-365; 146(1962), 195-216. http://dx.doi.org/10.1007/BF01470950

[23] $\longrightarrow$ The Levi problem in the theory of functions of several complex variables. Proc. Internat. Congr. Mathematicians (Stockholm, 1962), Inst. Mittag-Leffler, Djurholm, 1963, pp. 385-388.

[24] A. Onishchik, Complex hulls of compact homogeneous spaces. Dokl. Akad. Nauk SSSR 130(1960), 726-729 (Russian); translation in Soviet Math. Dokl. 1(1960), 88-91.

[25] R. Remmert, Sur les espaces analytiques holomorphiquement séparables et holomorphiquement convexes. C. R. Acad. Sci. Paris 243(1956), 118-121.

[26] J.-P. Serre, Quelques problèmes globaux relatifs aux variétés de Stein. In: Colloque sur les fonctions de plusieurs variables, tenu à Bruxelles, 1953, Georges Thone, Liège; Masson \& Cie, Paris, 1953, pp. 57-68.

[27] J. Tits, Espaces homogènes complexes compacts. Comment. Math. Helv. 37(1962/1963), 111-120. http://dx.doi.org/10.1007/BF02566965

Dept. of Mathematics \& Statistics, University of Regina, Regina, S4S OA2

e-mail: gilligan@math.uregina.ca 